\title{
Computational fluid dynamics analysis of tandem carotid artery stenoses: Investigation of neurological complications after carotid artery stenting
}

\author{
Yukinao Kambayashi ${ }^{a}$, Hiroyuki Takao ${ }^{\mathrm{a}, \mathrm{b}, \mathrm{e}, *}$, Kouichi Shinohara $^{\mathrm{b}}$, Takashi Suzuki ${ }^{\mathrm{b}}$, \\ Sho Takayama ${ }^{\mathrm{b}}$, Soichiro Fujimura ${ }^{\mathrm{b}}$, Shunsuke Masuda ${ }^{\mathrm{a}}$, Mituyoshi Watanabe ${ }^{\mathrm{a}}$, \\ Tomoaki Suzuki $^{\mathrm{a}}$, Chihebeddine Dahmani ${ }^{\mathrm{a}, \mathrm{c}}$, Toshihiro Ishibashi ${ }^{\mathrm{a}}$, Makoto Yamamoto $^{\mathrm{d}}$ and \\ Yuichi Murayama ${ }^{\mathrm{a}}$ \\ ${ }^{a}$ Department of Neurosurgery, The Jikei University School of Medicine, Tokyo, Tokyo, Japan \\ ${ }^{\mathrm{b}}$ Graduate School of Mechanical Engineering, Tokyo University of Science, Tokyo, Japan \\ ${ }^{\mathrm{c}}$ Siemens Healthcare K.K., Tokyo, Japan \\ ${ }^{\mathrm{d}}$ Department of Mechanical Engineering, Tokyo University of Science, Tokyo, Japan \\ ${ }^{\mathrm{e}}$ Department of Innovation for Medical Information Technology, Jikei University School of Medicine, \\ Tokyo, Japan
}

Received 14 February 2016

Accepted 23 April 2016

\begin{abstract}
.
BACKGROUND: Combined extra- and intracranial carotid artery stenoses, particularly involving multiple lesions, show complex hemodynamic properties and represent a therapeutic dilemma. We used computational fluid dynamics (CFD) to investigate whether insufficient cerebral blood flow (CBF) in a 70-year-old man with tandem stenoses was the cause of aphasia and right hemiparesis after carotid artery stenting (CAS) of the extracranial stenosis.

METHOD: Three-dimensional digital subtraction angiography (3D-DSA) was performed before and after balloon angioplasty and CAS in the patient. The geometrical and rheological conditions of the carotid arteries were determined, and computational meshes were generated from the patient-specific 3D-DSA datasets. CFD analysis was performed, and hemodynamic parameters such as mass flow, pressure, fractional flow reserve, and streamlines were calculated.

RESULTS: Post-CAS simulations showed that the percentage of internal carotid artery mass flow from common carotid artery mass flow increased from $9 \%$ to $14 \%$ and $\mathrm{CBF}$ improved by only $5 \%$.

CONCLUSIONS: CFD analysis suggested that the neurological complications were caused by insufficient CBF rather than embolic events, and in tandem carotid stenoses, CAS for an extracranial lesion alone may not always sufficiently increase CBF. CFD enabled the noninvasive quantitative estimation of the effects of CAS of each stenotic segment on carotid flow.
\end{abstract}

Keywords: Carotid artery stenting, carotid stenosis, cerebral blood flow, computational fluid dynamics, neurological complication

\footnotetext{
${ }^{*}$ Corresponding author: Hiroyuki Takao, Department of Neurosurgery, Jikei University School of Medicine Hospital, 3-25-8 Nishishinbashi, Minato-ku, Tokyo 105-0003, Japan. Tel.: +81 033433 1111; Fax: +81 033433 1111; E-mail: takao@jikei.ac.jp.

0928-7329/16/\$35.00 (c) 2016 - IOS Press and the authors. All rights reserved

This article is published online with Open Access and distributed under the terms of the Creative Commons Attribution NonCommercial License (CC BY-NC 4.0).
} 


\section{Introduction}

Carotid artery stenting (CAS) has been performed to prevent stroke in patients with carotid artery stenosis, and its efficacy has been previously validated [1,2]. However, some studies have shown that patients with multiple tandem stenotic segments were at a higher risk of ischemic stroke than those with only a single stenotic segment [3-5]. Combined extra- and intracranial stenoses, particularly involving multiple lesions, show complex hemodynamic properties and represent a therapeutic dilemma for clinicians. The purpose of this study was to use computational fluid dynamics (CFD) to investigate whether insufficient blood flow was the cause of neurological complications in a patient with extra- and intracranial stenoses who developed aphasia and right hemiparesis soon after CAS.

\section{Case presentation}

This study was approved by the ethics committee of the Jikei University School of Medicine. A 70year-old man complaining of occasional dysarthria and right-sided weakness for the previous 2 years presented to our outpatient clinic. Physical examination revealed no neurological deficits. The only significant medical history was hypertension, for which he was not taking any medications. Brain magnetic resonance imaging (MRI) showed no marked ischemic lesions, and carotid computed tomographic angiography showed 70\% left extracranial carotid stenosis (Fig. 1A), according to the North American Symptomatic Carotid Endarterectomy Trial criteria [2], and an additional 75\% intracranial carotid stenosis at the cavernous portion (Fig. 1B and C), according to the Warfarin-Aspirin Symptomatic Intracranial Disease study criteria [6]. A study using N-isopropyl-p- $\left[{ }^{123} \mathrm{I}\right]$ iodoamphetamine single-photon emission computed tomography (SPECT) showed low cerebral blood flow (CBF) on the left side (Table 1). Transient ischemic attacks and symptomatic carotid stenoses were diagnosed on the basis of these clinical findings.

Therefore, CAS was performed for the left extracranial carotid stenosis (Fig. 1D). The procedures were performed via femoral access under local anesthesia and systemic heparinization for maintaining an activated clotting time of $>200 \mathrm{~s}$. A 6-Fr shuttle sheath was placed at the distal common carotid artery. The carotid lesion was crossed using FilterWire EZ ${ }^{\mathrm{TM}}$ (Boston Scientific, Natick, MA, USA) that was deployed at the distal internal carotid artery (ICA), with pre-dilation (6 atm) performed using an undersized angioplasty balloon (Sterling, $3.0 \times 40 \mathrm{~mm}$; Boston Scientific). An appropriately sized stent (Carotid Wallstent, $10 \times 31 \mathrm{~mm}$; Boston Scientific) was deployed, followed by post-dilation $(6$ atm) using an appropriately sized balloon (Sterling, $4.0 \times 40 \mathrm{~mm}$; Boston Scientific). After CAS, the patient was admitted to the intensive care unit for close observation, and his systolic blood pressure was maintained within the range of $120-160 \mathrm{mmHg}$ to avoid the hyperperfusion syndrome.

However, approximately $3 \mathrm{~h}$ after CAS, he developed aphasia and right hemiparesis. Brain MRI, including diffusion-weighted imaging, was performed, but it was uninformative and revealed no acute ischemic lesions.

The patient's symptoms continued the next day; therefore, MRI and cerebral angiography were repeated. Findings from both were not markedly different from those before CAS. For further evaluation, SPECT was performed, which showed continued low CBF on the left side, despite the treatment (Fig. 1, Table 1). As a result, the symptoms were attributed to hemodynamic compromise rather than embolism. It was postulated that this hemodynamic compromise was caused by intracranial stenosis and inadequate blood pressure control, which led to relatively lower values than the preoperative values. Balloon angioplasty (6 atm) (Gateway M/R, $2.0 \times 9 \mathrm{~mm}$; Boston Scientific) was performed for intracranial carotid 
Table 1

SPECT left/right CBF ratio

\begin{tabular}{lccc}
\hline Left/right CBF ratio & Pre-CAS & Post-CAS & Post-treatment \\
\hline Pericallosal & 0.96 & 0.92 & 0.96 \\
Callosomarginal & 0.91 & 0.93 & 0.95 \\
Precentral & 0.86 & 0.79 & 0.93 \\
Central & 0.94 & 0.82 & 0.95 \\
Parietal & 0.91 & 0.80 & 1.00 \\
Angular & 0.87 & 0.73 & 1.04 \\
Temporal & 0.91 & 0.76 & 0.98 \\
\hline
\end{tabular}

CBF, cerebral blood flow; SPECT, single-photon emission computed tomography; CAS, carotid artery stenting.

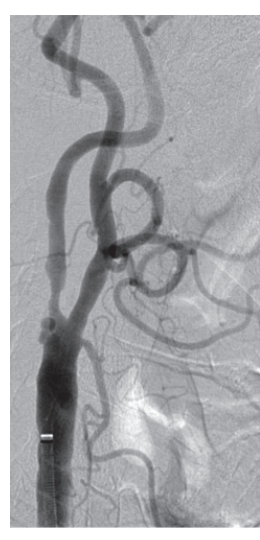

(240 pixel x 519 pixel)

A

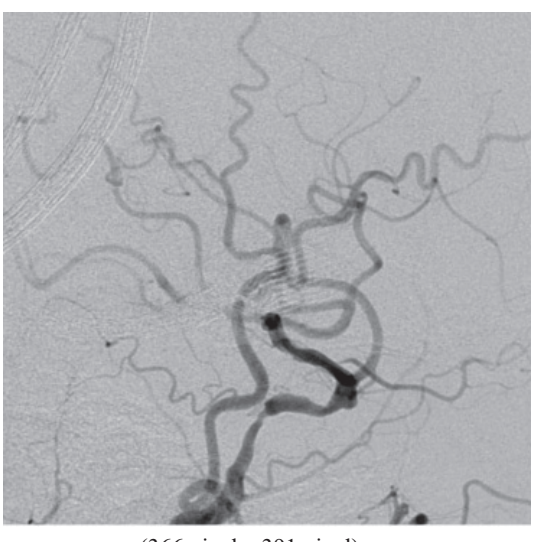

(366 pixel x 391 pixel)

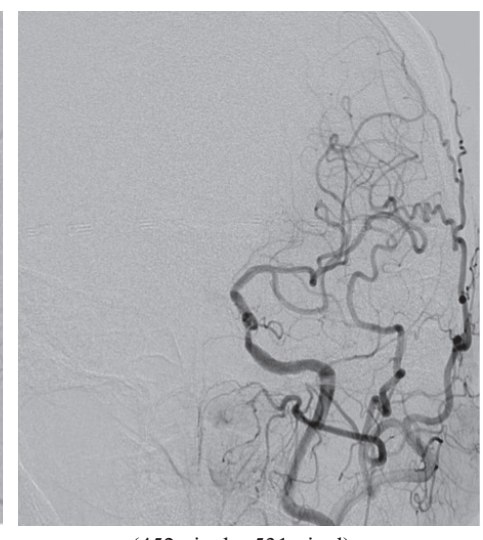

(452 pixel x 531 pixel)

$\mathrm{C}$

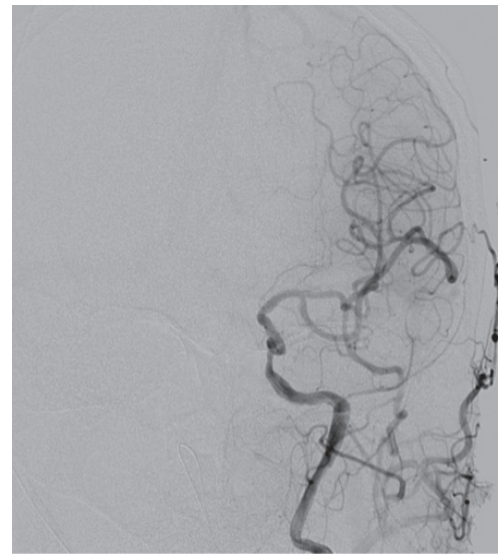

(446 pixel x 531 pixel)

G

Fig. 1. DSA of the carotid artery. Left extracranial carotid stenosis (A), intracranial carotid stenosis (B), intracranial flow from the left common carotid artery before CAS (C), and carotid artery and intracranial flow immediately after CAS (D and E) and on the next day (F and G). DSA, digital-subtraction angiography; CAS, carotid artery stenting.

stenosis. Blood pressure was maintained within the range of 140-160 mmHg. Subsequently, the symptoms resolved, and the patient was discharged 7 days later with a modified Rankin scale score of 0 . The follow-up SPECT showed CBF improvement on the left side (Table 1). 
Table 2

Mass flow ICA/CCA and FFR

\begin{tabular}{lcc}
\hline & Mass flow ICA/CCA $(\%)$ & FFR $(\%)$ \\
\hline Pre-CAS & 9 & 4 \\
Post-CAS & 14 & 40 \\
Normal adults & 63 & $\geqq 80$ \\
\hline
\end{tabular}

ICA, internal carotid artery; CCA, common carotid artery; FFR, fractional flow reserve; CAS, carotid artery stenting.

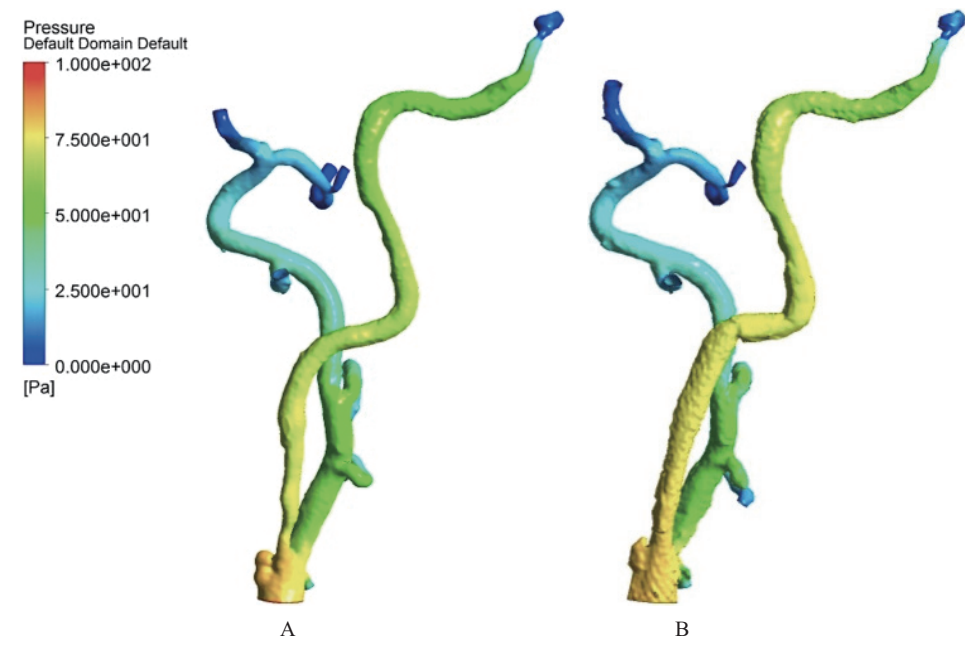

Fig. 2. Pressures before (A) and after CAS (B). CAS, carotid artery stenting.

\subsection{CFD analysis}

Three-dimensional digital-subtraction angiography was performed before and after the deployment of the carotid stent. The geometry of the carotid artery was reconstructed, extracted by manual cropping, and converted into a triangulated surface (Real Intage; Cybernet Systems, Tokyo, Japan). An unstructured computational volumetric mesh was constructed from the triangulated surface. This mesh mainly comprised $4 \times 10^{6}$ tetrahedrons with multiple layers of prism elements placed near the wall surface to increase the analytical precision of the boundary layer. Blood flow simulations were performed using ANSYS ICEM CFD (ANSYS; Canonsburg, PA, USA) based on Navier-Stokes equations in a homogeneous, Newtonian, viscous fluid with a density of $1100 \mathrm{~kg} / \mathrm{m}^{3}$ and a viscosity of $0.0036 \mathrm{~Pa}$ under non-pulsatile flow conditions at Reynolds number around 500. Boundary conditions were set as follows on the basis of the data for older adults [7]: for the inlet, mass flow of $0.003153 \mathrm{~kg} / \mathrm{s}$, and for the outlet, opening of $0 \mathrm{~Pa}$, Hemodynamic parameters such as mass flow, pressure, fractional flow reserve (FFR), and streamlines were calculated based on convergence criteria; Root Mean Square (RMS) (Rediual Target; 0.0001). FFR is the ratio of pressure distal to the lesion to pressure proximal to the lesion.

\section{Results}

Blood flow simulations were successfully performed. Mass flow, pressure, FFR and streamlines were calculated (Figs 2, 3, Table 2). The results demonstrated that the percentage of ICA mass flow from that of the common carotid artery increased from $9 \%$ to only $14 \%$ after balloon angioplasty and deployment 


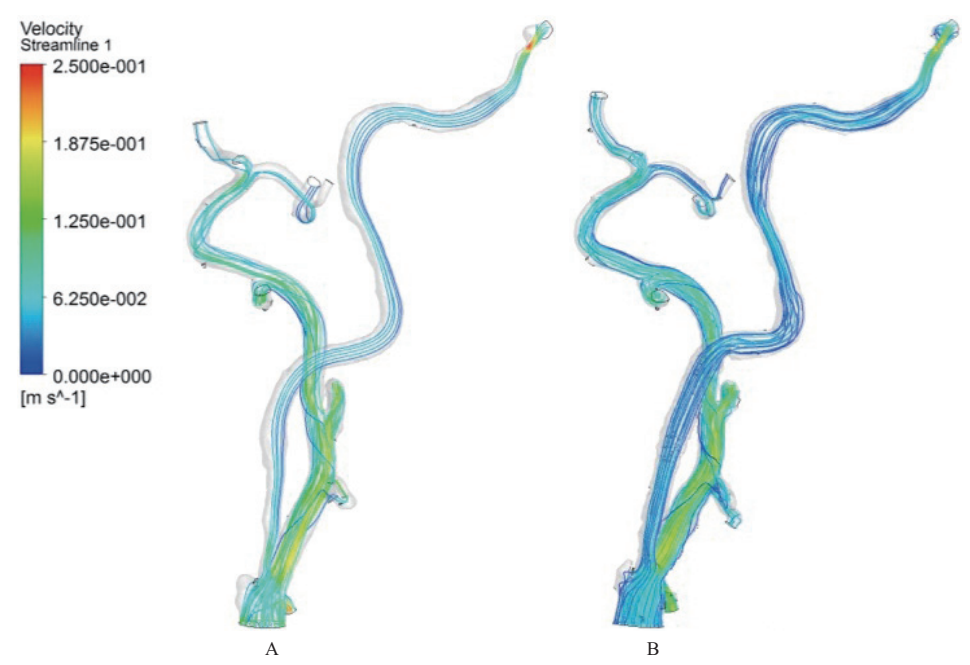

Fig. 3. Streamlines before (A) and after CAS (B). CAS, carotid artery stenting.

of the carotid stent. In both simulations, low pressure was observed, particularly at the clinoidal portion of ICA, just distal to the intracranial stenosis. FFR also increased from $4 \%$ to $40 \%$.

\section{Discussion}

Although CAS has been established as a safe technique, some periprocedural complications associated with hemodynamic changes remain of concern, and hemodynamic instability is not an infrequent phenomenon after this procedure $[1,2,8]$. In the present case, the improvement of mass flow was only $5 \%$ even after stent deployment. This as well as the SPECT findings suggested that the neurological complications after CAS were caused by insufficient CBF. In addition to investigating the causes of complications, CFD analysis based on patient-specific data can be used to estimate blood flow at each segment. During the computational simulation of basic vessel models containing two stenotic lesions, variations in CBF improvement have been reported when treatment for the proximal lesion is performed [9]. CBF improvement is less in cases with the severity of the proximal lesion almost the same as that of the distal lesion in comparison with the in cases with the severity of proximal lesion higher than that of the distal lesion [9]. Our findings are consistent with these results, and the improvement after treatment of the extracranial stenosis appeared insufficient to recover the required CBF. Therefore, during carotid stenosis, we should evaluate each stenotic segment separately when multiple lesions are identified and then decide on the treatment with a clear understanding of the contribution of each stenotic segment toward flow reduction.

Hemodynamic changes are often estimated using perfusion imaging modalities such as SPECT and positron emission tomography $[10,11]$. In the present case, SPECT was effective for investigating the causes of the neurological complications. However, these imaging modalities evaluate regional CBF and have some limitations in evaluating the CBF provided exclusively via the carotid system. CFD is a tool for simulating various situations based on patient-specific data. In the field of cardiology, CFD analysis has been validated for the evaluation of stenotic lesions [12]. In coronary artery stenosis, a fractional flow reserve (FFR) value of $<80 \%$ is used as the criterion for endovascular treatment [13]. Because there was no validated value for FFR, it could not be used as a criterion for the indications for management. 
However, we may consider it as an expression of insufficient flow, given the clinical and hemodynamic conditions. Further validation of significant FFR values can be a valuable aid in decision-making related to the effective management of tandem and carotid stenoses in general.

\section{Conclusion}

The study findings in our case of "tandem" (extra-intracranial) carotid stenoses suggested that CAS alone for extracranial stenosis is not always effective for sufficiently increasing CBF. CFD analysis is a noninvasive tool that is effective for evaluating hemodynamic changes and quantitatively estimating the effects of CAS treatment of each stenotic segment on carotid flow.

\section{Acknowledgments}

This work was partially supported by a research grant from Siemens Healthcare K.K. provided to our academic institution for this study. Siemens Healthcare K.K. had no involvement in this study.

\section{Conflict of interest}

C.D. is a full-time employee of Siemens Healthcare K.K. The other authors have no conflicts of interest to disclose.

Y.M. and T.I. have received honoraria from Stryker Japan Y.M. has received honoraria from Asahi Intecc Co., Ltd.

Y.M. and H.T. were partially supported by Siemens Healthcare K.K. with a grant provided to our academic institution (Grant No. 35993-00211563).

H.T. was supported by NTT DOCOMO, INC. with a donation provided to our academic institution outside the submitted work.

\section{References}

[1] Silver FL, Mackey A, Clark WM, Brooks W, Timaran CH, Chiu D, et al. Safety of stenting and endarterectomy by symptomatic status in the Carotid Revascularization Endarterectomy Versus Stenting Trial (CREST). Stroke. 2011; 42(3): 675-680. doi: 10.1161/STROKEAHA.110.610212.

[2] Njemanze PC, Beck OJ, Gomez CR, Horenstein S, Cujec B, Polasek P, et al. North American Symptomatic Carotid Endarterectomy Trial. Methods, patient characteristics, and progress. Stroke. 1991; 22(6): 711-720.

[3] Marzewski DJ, Furlan AJ, St Louis P, Little JR, Modic MT, Williams G. Intracranial internal carotid artery stenosis: longterm prognosis. Stroke. 1982; 13(6): 821-824.

[4] Siddiqui FM, Hassan AE, Tariq N, Yacoub H, Vazquez G, Suri MF, et al. Endovascular management of symptomatic extracranial stenosis associated with secondary intracranial tandem stenosis. A multicenter review. J Neuroimaging. 2012; 22(3): 243-248. doi: 10.1111/j.1552-6569.2011.00611.x.

[5] Stelagowski M, Bogusiak K, Kasielska A, Łysakowski M, Kaźmierski P, Szostek M. Intracranial occlusions and internal carotid artery stenoses: clinical implications. Ann Vasc Surg. 2010; 24(6): 786-793. doi: 10.1016/j.avsg.2010.02.033.

[6] Chimowitz MI, Kokkinos J, Strong J, Brown MB, Levine SR, Silliman S, et al. The Warfarin-Aspirin Symptomatic Intracranial Disease Study. Neurology. 1995; 45(8): 1488-1493.

[7] Hoi Y, Wasserman BA, Xie YJ, Najjar SS, Ferruci L, Lakatta EG, et al. Characterization of volumetric flow rate waveforms at the carotid bifurcations of older adults. Physiol Meas. 2010; 31(3): 291-302. doi: 10.1088/0967-3334/31/3/002.

[8] Mylonas SN, Moulakakis KG, Antonopoulos CN, Kakisis JD, Liapis CD. Carotid artery stenting-induced hemodynamic instability. J Endovasc Ther. 2013; 20(1): 48-60. doi: 10.1583/12-4015.1. 
[9] Li ZY, Taviani V, Tang T, Sutcliffe MP, Gillard JH. The hemodynamic effects of in-tandem carotid artery stenosis: implications for carotid endarterectomy. J Stroke Cerebrovasc Dis. 2010; 19(2): 138-145. doi: 10.1016/j.jstrokecere brovasdis.2009.03.014.

[10] Horie N, Kitagawa N, Morikawa M, Kaminogo M, Nagata I. Monitoring of regional cerebral oxygenation by nearinfrared spectroscopy in carotid arterial stenting: preliminary study. Neuroradiology. 2005; 47(5): 375-379. doi: 10.1007/ s00234-004-1326-8.

[11] Matsubara S, Moroi J, Suzuki A, Sasaki M, Nagata K, Kanno I, Miura S. Analysis of cerebral perfusion and metabolism assessed with positron emission tomography before and after carotid artery stenting. J Neurosurg. 2009; 111(1): 28-36. doi: 10.3171/2008.09.17663.

[12] Taylor CA, Fonte TA, Min JK. Computational fluid dynamics applied to cardiac computed tomography for noninvasive quantification of fractional flow reserve: scientific basis. J Am Coll Cardiol. 2013; 61(22): 2233-2241. doi: 10.1016/j.jacc.2012.11.083.

[13] Tonino PA, De Bruyne B, Pijls NH, Siebert U, Ikeno F, van't Veer M, et al. Fractional flow reserve versus angiography for guiding percutaneous coronary intervention. N Engl J Med. 2009; 360(3): 213-224. doi: 10.1056/NEJMoa0807611. 